\title{
Ectodermal Dysplasia with Anodontia: A Report of Two Cases
}

\author{
Mehmet Bania \\ Ali Melih Tezkirecioglu \\ Nese Akalc \\ Tamer Tuzunerd
}

\section{ABSTRACT}

Ectodermal dysplasia is a hereditary disorder that occurs as a consequence of disturbances in the ectoderm of the developing embryo. The triad of nail dystrophy, alopecia or hypotrichosis and palmoplantar hyperkeratosis is usually accompanied by a lack of sweat glands and a partial or complete absence of primary and/or permanent dentition. Two case reports illustrating the prosthetic rehabilitation of 2 young boys with anhidrotic ectodermal dysplasia associated with severe anodontia are presented. Since the oral rehabilitation of these cases is often difficult; particularly in pediatric patients, treatment should be administered by a multidisciplinary team involving pediatric dentistry, orthodontics, prosthodontics and oral-maxillofacial surgery. (Eur J Dent 2010;4:215-222)

Key words: Ectodermal dysplasia; Anodontia; Prosthetic rehabilitation; Multidisciplinary management.

- aDS, Research Assistant, Gazi University, Faculty of Dentistry, Department of Paediatric Dentistry, Ankara, Turkey.

b DDS, PhD, Research Assistant, Gazi University, Faculty of Dentistry, Department of Paediatric Dentistry, Ankara, Turkey.

DDS, PhD, Professor, Gazi University, Faculty of Dentistry, Department of Paediatric Dentistry, Ankara, Turkey.

d DDS, PhD, Assistant Professor, Karadeniz Technical University, Faculty of Dentistry, Department of Paediatric Dentistry, Trabzon, Turkey.

- Corresponding author: Dr. Mehmet Bani Gazi Universitesi, Dis Hekimligi Fakultesi, Pedodonti Anabilim Dali

8. Cad. 82. sok 06510 Emek, Ankara, Turkey.

Phone: +90312 2034090

Fax: +903122034062

E-mail:mehmetbaniahotmail.com mehmetbanidgazi.edu.tr

\section{INTRODUCTION}

Ectodermal dysplasia, as first described by Thurman, ${ }^{1,2}$ is a hereditary disorder occurring as a consequence of disturbances in the ectoderm of the developing embryo. The triad of nail dystrophy (onchodysplasia), alopecia or hypotrichosis (scanty, fine light hair on the scalp and eyebrows), and palmoplantar hyperkeratosis is usually accompanied by a lack of sweat glands (hypohidrosis) and a partial or complete absence of primary and/or permanent dentition. ${ }^{2-5}$

Ectodermal dysplasia represents a large and complex group of diseases comprising more than 170 different clinical conditions. ${ }^{3}$ The incidence of this condition is $1: 100,000$, with a mortality rate of $28 \%$ in males up to 3 years of age. ${ }^{1}$ When at least 2 types of abnormal ectodermal features occur, 
such as malformed teeth and extremely sparse hair, the patient is diagnosed with ectodermal dysplasia syndrome. ${ }^{3,5}$

There are 2 major types of this condition depending on the number and functionality of the sweat glands: (1) X-linked anhidrotic or hypohidrotic, where sweat glands are either absent or significantly reduced in number (Christ-SiemensTouraine syndrome), and (2) hidrotic, where sweat glands are normal and the condition is inherited as autosomal dominant (Clouston's syndrome). 1,3,5 The dentition and hair are affected similarly in both types, but the hereditary patterns and nail and sweat gland manifestations tend to differ. ${ }^{2}$

Christ-Siemens-Touraine syndrome, with $X$-linked recessive inheritance, is the most frequently reported manifestation of ectodermal dysplasia. , 7,8 Depending on the severity of clinical manifestations, Christ-Siemens-Touraine syndrome can be classified as either hypohidrotic or anhidrotic ectodermal dysplasia. ${ }^{8}$

Oral traits of ectodermal dysplasia (ED) may be expressed as anodontia or hypodontia, with or without a cleft lip and palate. Anodontia also manifests itself by a lack of alveolar ridge development; ${ }^{7}$ as a result, the vertical dimension of the lower face is reduced, the vermilion border disappears, existing teeth are malformed, the oral mucosa becomes dry, and the lips become prominent. The face of an affected child usually has the appearance of old age..$^{7-9}$

Genetic studies regarding the etiology of ED reveal that mutations in the ectodysplasin- $A$ and ectodysplasin-A receptor genes are responsible for X-linked and autosomal hypohidrotic ectodermal dysplasia. ${ }^{2}$ The clinical diagnosis is based on clinical and radiological manifestations such as the number and distribution of sweat pores, the amount of sweat produced, the structural and biochemical characteristics of hair, skin biopsy, and characteristics of lacrimal secretions. ${ }^{10}$ Alopecia areata, incontinentia pigmenti, Werner syndrome, focal dermal hypoplasia, familial simple anhidrosis, and dyskeratosis congenita are some of the conditions through which the differential diagnosis is made. ${ }^{11}$

The most frequent prosthetic treatment for the dental management of ectodermal dysplasia is removable prosthodontics. Since alveolar bone development is dependent on the presence of teeth, children with ectodermal dysplasia have little or no bone ridge upon which to construct dentures; therefore, restoring function and appearance is more challenging than usual. ${ }^{3}$ Follow-up by a multidisciplinary team involving pediatric dentistry, orthodontics, prosthodontics, and oral-maxillofacial surgery specialists is advocated to be the most appropriate approach in such cases. ${ }^{8}$ In this case report; it is aimed to describe the prosthetic rehabilitation of 2 young boys with anhidrotic ectodermal dysplasia associated with severe anodontia.

\section{CASE REPORT}

\section{Case 1}

An 8-year old male patient was referred to Gazi University Faculty of Dentistry Department of Pediatric Dentistry due to lack of teeth as well as speech and mastication problems. Parental history revealed that the patient was diagnosed with anhidrotic ectodermal dysplasia after skin biopsy at Gazi University Faculty of Medicine Department of Pediatrics 5 years ago. The patient was the only child and there were no other cases of ectodermal dysplasia in the family. The lack of primary and permanent teeth in the oral cavity resulted in dietary problems; in addition, the child did not accept any previous dental intervention due to anxiety.

During extraoral examination, the child exhibited the typical features of anhidrotic ectodermal dysplasia: saddle nose; soft, dry and light colored skin; increased pigmentation; as well as thin, linear wrinkles in the peri-oral region (Figures 1 and 2). Intraoral examination revealed the complete absence of primary and permanent teeth, thin alveolar ridges, reduced vertical bone height, and loss of sulcus depth in the posterior regions of maxillary and mandibular jaws (Figures 3 and 4); complete anodontia was also confirmed by panoramic radiography (Figure 5). In order to improve appearance, mastication, and speech, removable complete maxillary and mandibular dentures were determined to be the best treatment choice.

Routine procedures were followed for the construction of the dentures. Preliminary impressions were made with irreversible hydrocolloid, and then custom trays were prepared for functional impression. Acrylic bases with wax rims were made on the master casts in order to establish maxil- 
lomandibular relations. After making the maxillomandibular records, the casts were mounted in an articulator. Rather than primary tooth forms, permanent tooth forms were selected in order to provide better static and dynamic occlusion. Primary tooth forms were not adequate to fulfill the ideal vertical dimensions because the patient is in the transition period from mixed to permanent dentition. After the final insertion, routine hygiene instructions for the dentures were given to both the child and his parents (Figures 6 and 7). The patient was advised to maintain a soft diet for the first few days, and to remove the dentures at night to promote healing of the oral tissue. Despite the initial lack of compliance, the child tolerated the dentures quite well. In order to accommodate growth and development, the patient was scheduled for ongoing follow up visits every 3 months. At recall appointments, good retention was observed and the parents reported a significant improvement in terms of speech and mastication; in addition, they

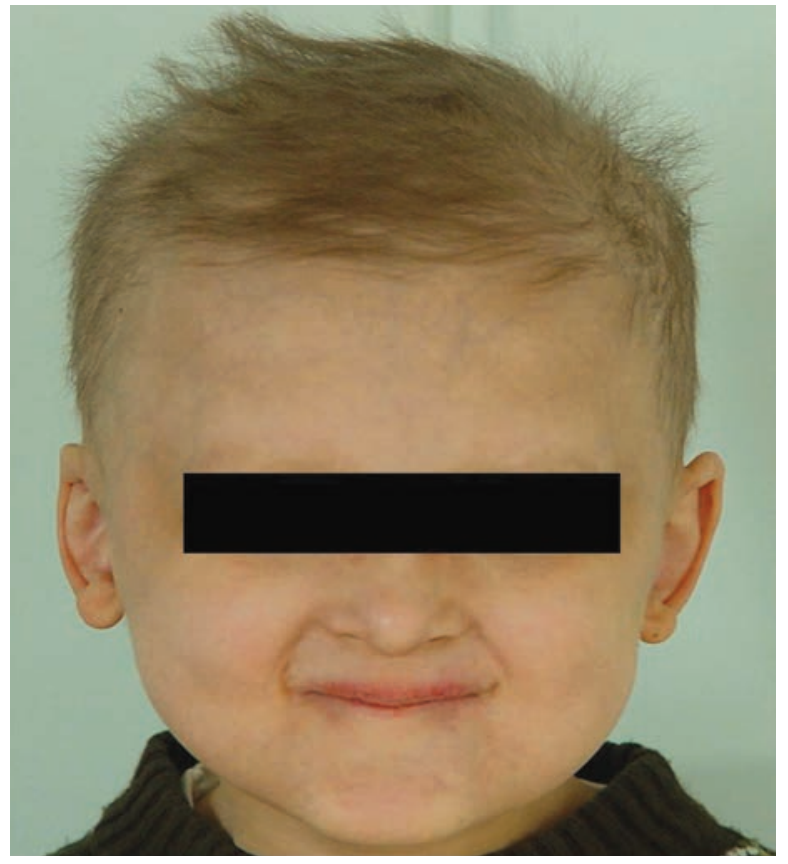

Figure 1. Facial view of the patient.

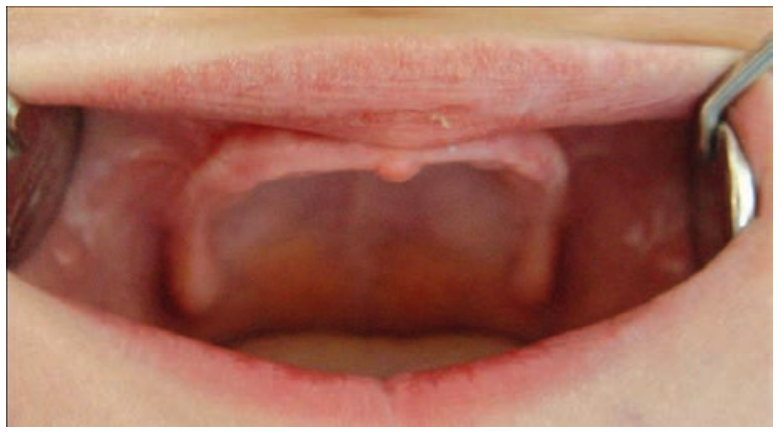

Figure 3. Intraoral view of the upper jaw. discovered that he enjoyed wearing the dentures. Continued follow-up is suggested for modification or replacement of the dentures to fit the patient's developing maxilla and mandible.

\section{Case 2}

A 3-year old male patient was referred to Gazi University Faculty of Dentistry Department of Pediatric Dentistry due to the lack of primary teeth. The major complaint of his parents was mastication issues that resulted in dietary deficiencies; the parents further reported that he was unable to speak.

In the clinical extraoral examination, prominent forehead, sparse, very fine scalp hair and eyebrows, saddle nose, protuberant lips, large chin, soft, dry and light colored skin could be observed (Figures 8 and 9). Intraoral examination revealed complete absence of primary and permanent teeth, underdeveloped maxillary and mandibular ridges and relatively enlarged tongue (Figures

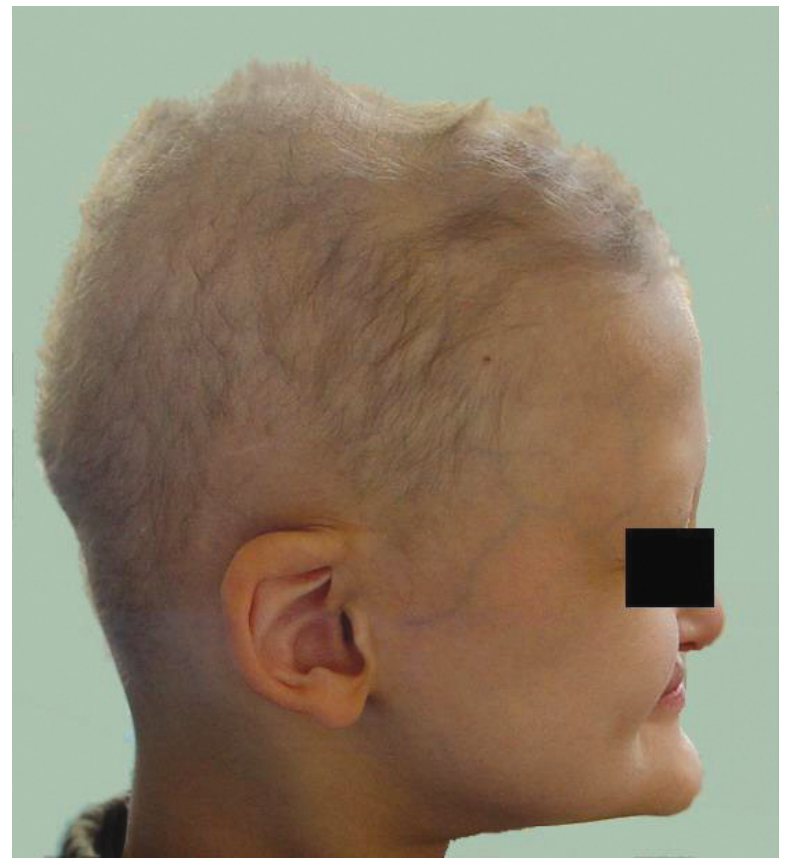

Figure 2. Profile view of the patient.

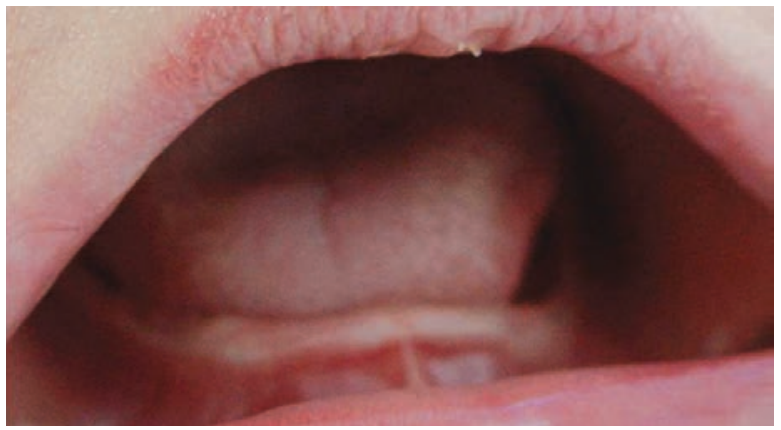

Figure 4 . Intraoral view of the lower jaw. 
10 and 11). Radiographic examination confirmed complete absence of both primary and permanent teeth as well as underdevelopment of alveolar ridges (Figure 12). Parental history revealed that there were no other cases of ectodermal dysplasia in his family. Since clinical findings suggest the presence of ectodermal dysplasia, the patient was referred to Gazi University Faculty of Medicine De- partment of Pediatrics. The patient was diagnosed anhidrotic ectodermal dysplasia by performing skin biopsy. To improve appearance, mastication and speech, it was decided that removable complete maxillary and mandibular dentures would be appropriate for the patient.

Dentures were fabricated following the same procedures described for the first case. Primary

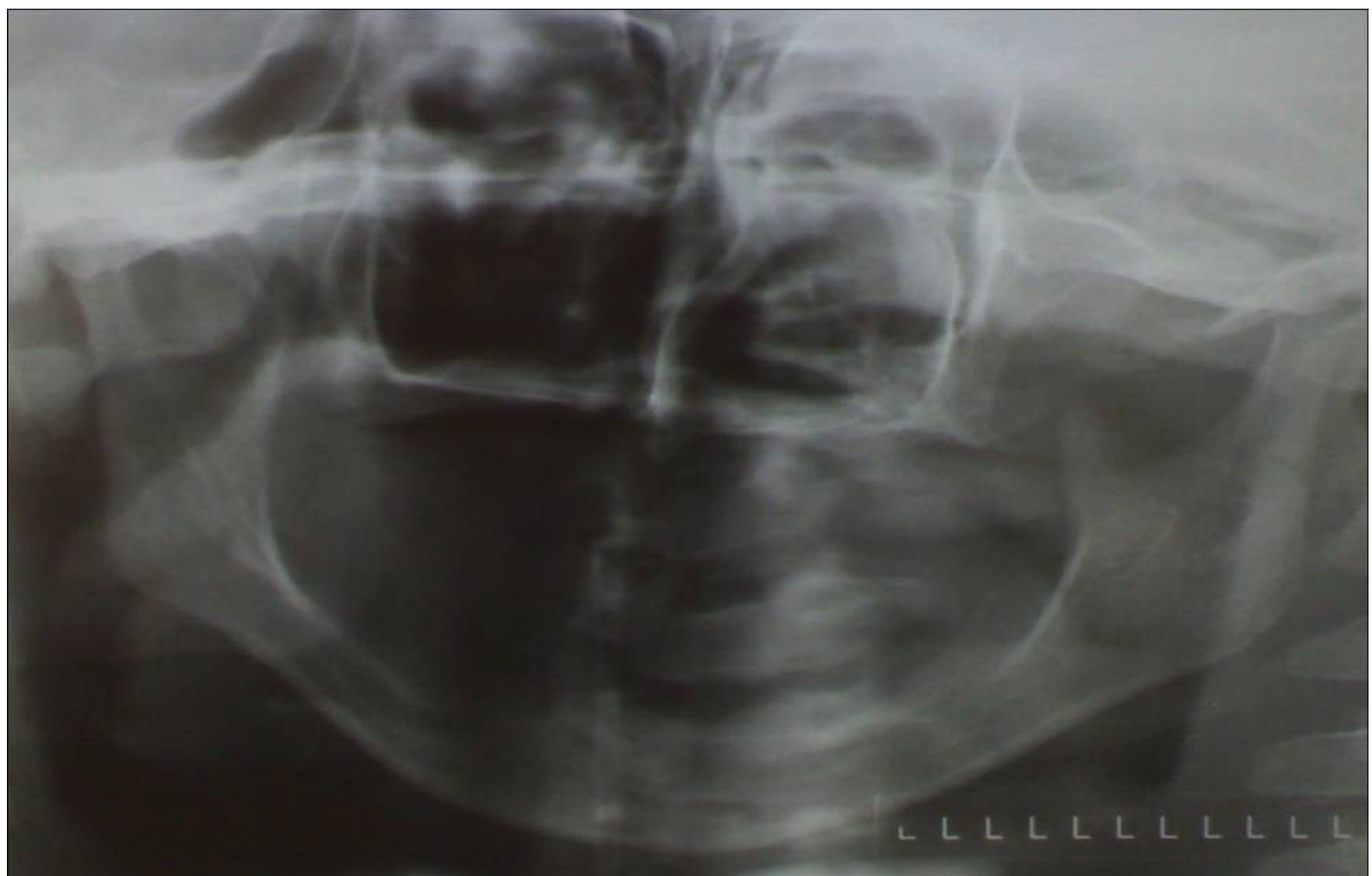

Figure 5. Panoramic radiograph confirming complete absence of teeth.

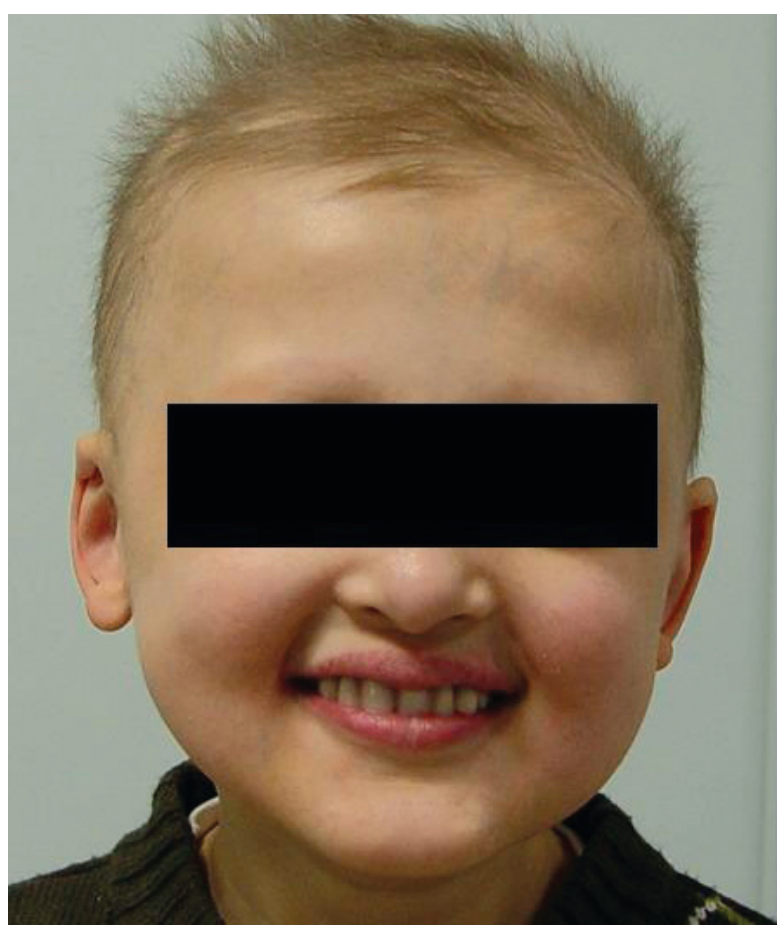

Figure 6. Facial view after treatment.

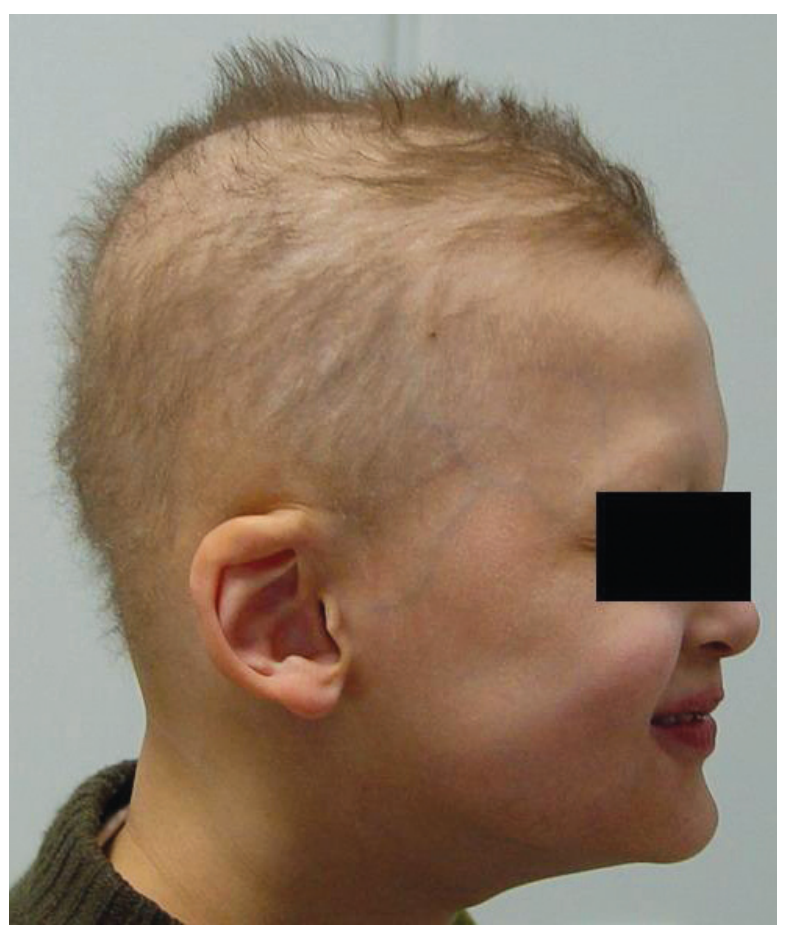

Figure 7. Profile view after treatment.

European Journal of Dentistry 
tooth forms were used and the occlusal vertical dimension was increased by $1 \mathrm{~mm}$ in order to improve the balance of both the dentures and facial profile. After the initial insertion, oral hygiene instructions for the dentures were given to the parents. Initially, the patient had some difficulty in accepting the dentures and was unable to keep them in his mouth due to his young age. After a few months, he was fully adapted to using the dentures, and his parents reported that he was able to eat; in addition, his speech improved and he was quite happy with the dentures (Figures 13, 14 and 15). Further follow-ups have taken place every 3 months. Further adjustments were made to eliminate interferences at recall appointments; future treatment will include relining, rebasing, or remaking the dentures in order to accommodate growth and development.

\section{DISCUSSION}

Oral rehabilitation of the ectodermal dysplasia patient is necessary to improve both the sagittal

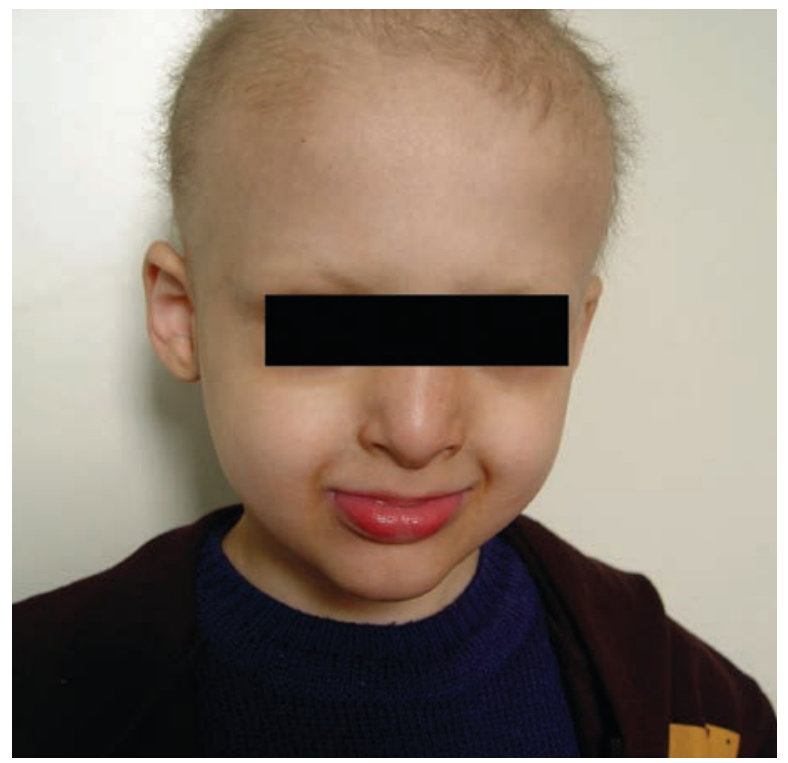

Figure 8. Facial view of the patient.

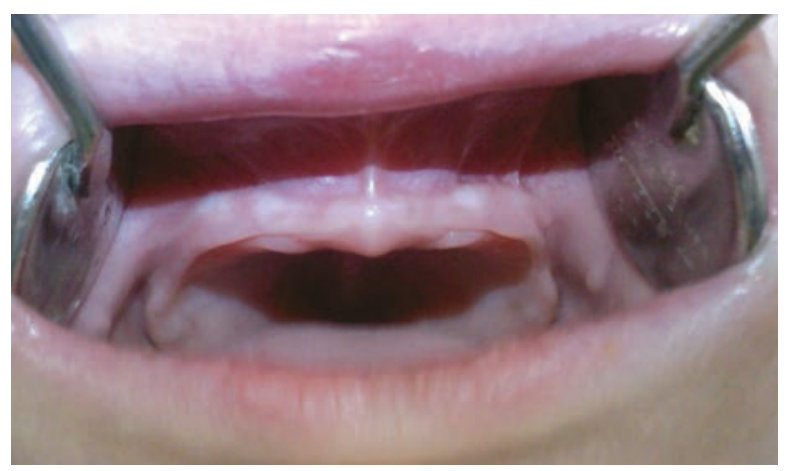

Figure 10. Intraoral view of the upper jaw. and vertical skeletal relationship during craniofacial growth and development as well as to provide improvements in esthetics, speech, and masticatory efficiency. ${ }^{2}$ Although removable prostheses are the most common treatment method, dental implants are also considered to be a treatment option. Dental implants combined with implantsupported dentures for adolescents over 12 years of age are recommended as a treatment choice in literature. In situations where implant therapy is indicated, the main problem is insufficient bone; if bone atrophy progresses in these already alveolar-deficient patients, implant placement may not be possible without bone grafting. ${ }^{7}$

Conversely, implantation reconstruction surgery is subject to a greater risk of failure compared to more conservative prosthetic treatment, besides its psychological aspects particularly in young children. .12 $^{2}$ Early implant placement in a growing child may cause cosmetic problems because the implants act like ankylosed teeth. With the vertical development of the jaws, implant

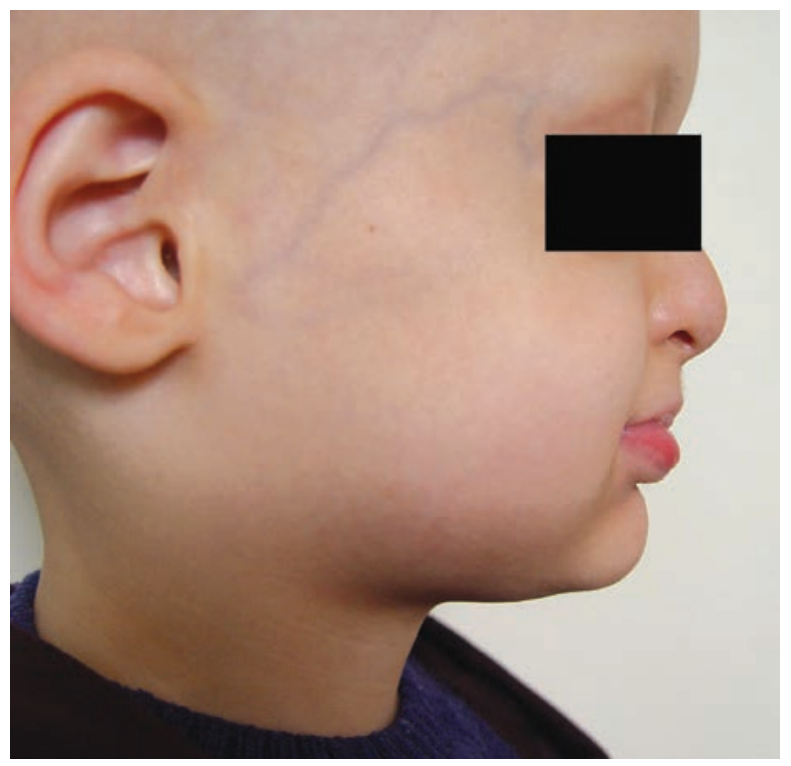

Figure 9. Profile view of the patient.

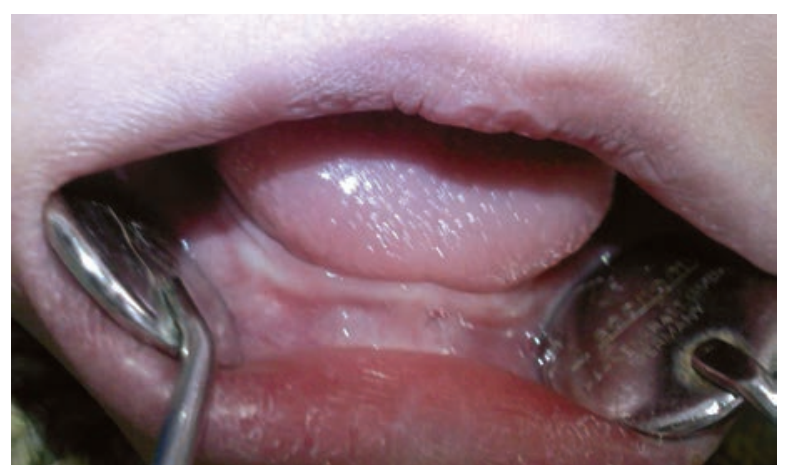

Figure 11. Intraoral view of the lower jaw. 
over-structures may not meet with the teeth of the opposite jaw, and may result in prosthetic infraocclusion. ${ }^{7.13}$ Therefore, the use of implants in young children should be considered carefully, taking into account the above-mentioned issues, especially dental and skeleton maturation as compared to the chronologic age of the patient. In both of the above cases, implant therapy was not the treatment choice due to ongoing growth and de- velopment and insufficient alveolar bone support.

It is well-known that dental findings in ectodermal dysplasia may range from hypodontia to anodontia of the primary or permanent teeth. However, the congenital absence of primary teeth is relatively rare; $5,9,10$ nevertheless, complete anodontia involving primary and permanent dentitions was observed in both cases. In the second case, the parents were unaware of their child's condi-

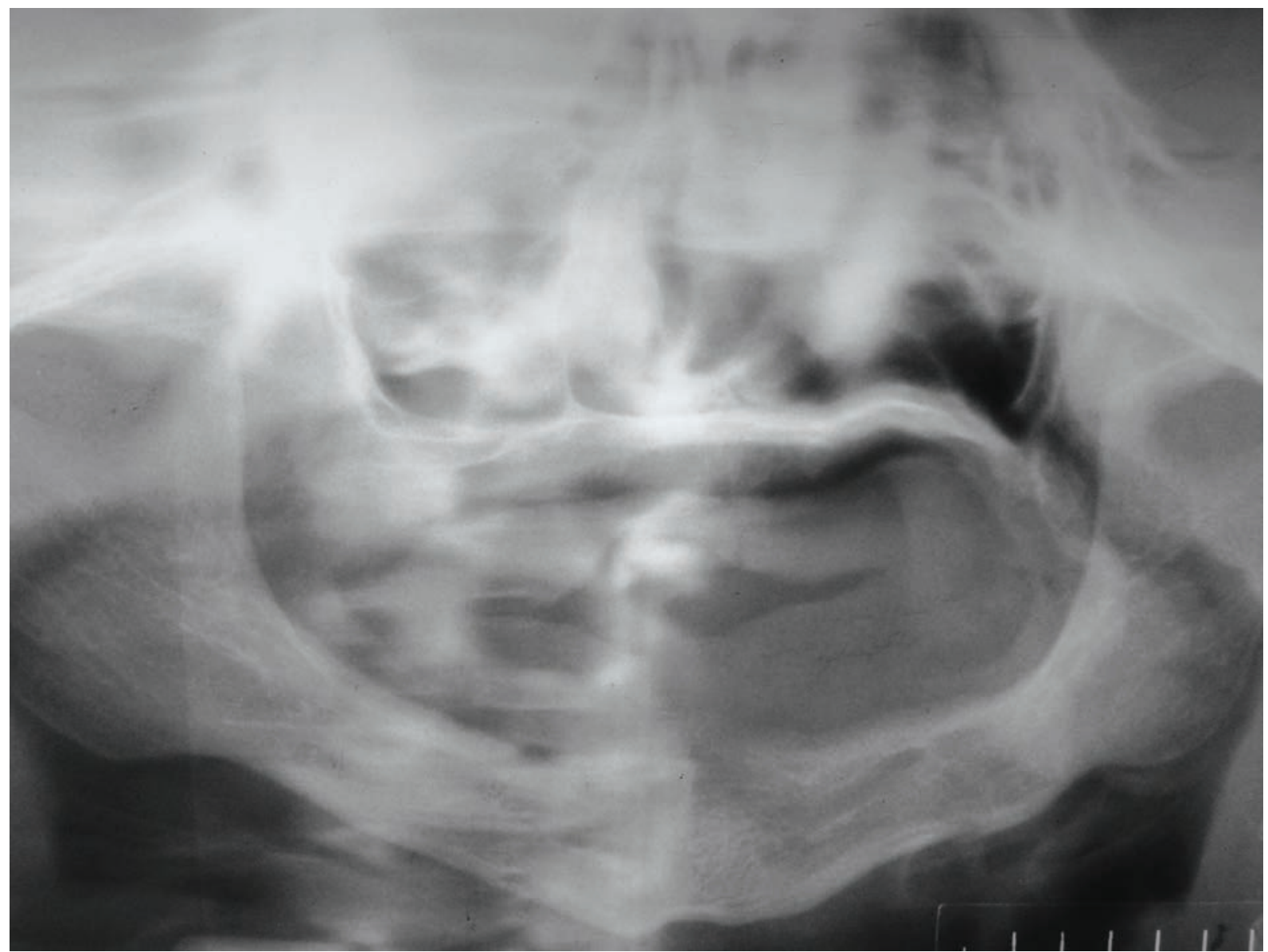

Figure 12. Panoramic radiograph confirming complete absence of teeth.

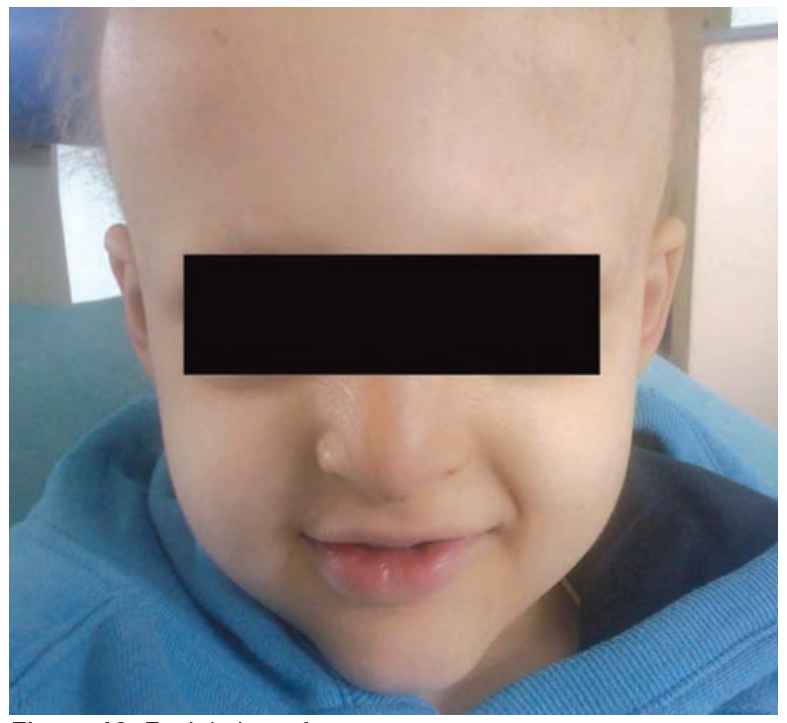

Figure 13. Facial view after treatment.

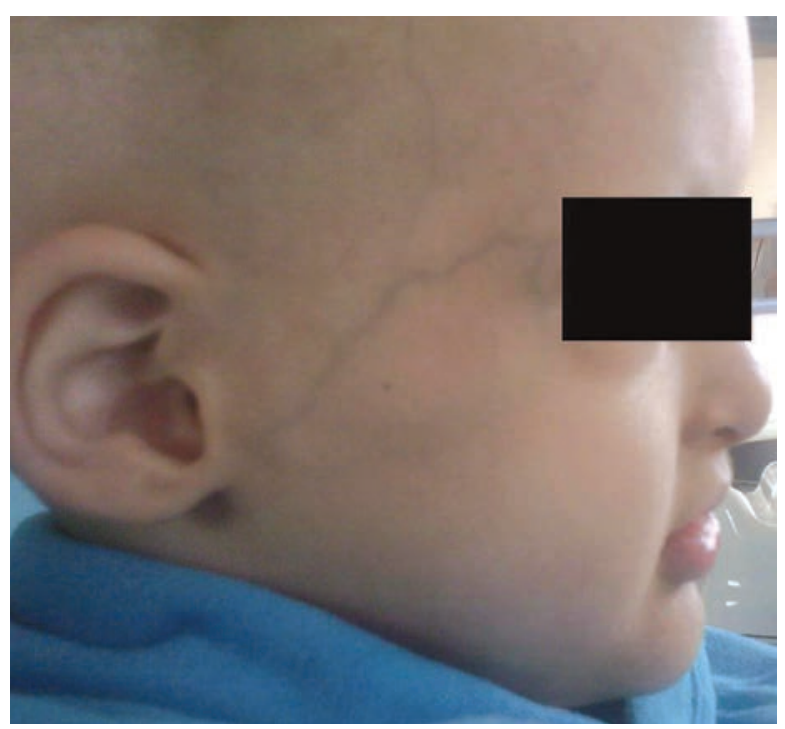

Figure 14. Profile view after treatment.

European Journal of Dentistry 
tion prior to a routine dental visit, after which anodontia and extra-oral findings were thought to be related to ectodermal dysplasia and the patient was then referred to a pediatrician.

There is little research regarding the impact of extensive hypodontia or anodontia on young children; ${ }^{14}$ it is known that from around 9 years of age, children with disabilities realize that they will remain different from other children. Therefore, the prosthetic intervention at the age of 8 in Case 1 is thought to be beneficial in terms of psychological development. Although the definite time to initialize treatment is still controversial, Till and Marquez ${ }^{15}$ recommend that an initial prosthesis could be fitted when the child starts school, so that he may enjoy a better appearance and will have time to adapt to the prosthesis. Due to the lack of compliance in case 1, the patient could not receive any dental treatment prior to his referral to our clinic. However, consequences of the delay in regards to speech, mastication, and psychological development were not determined to be significant. After using appropriate behavior management techniques, it was eventually possible to achieve prosthetic treatment.

In case 2, early prosthetic treatment led to significant improvements in appearance, speech, and masticatory function. During the first month following the initiation of prosthetic treatment, it was difficult for the 3-year old patient to adapt to the complete dentures; however, he was accustomed to using the dentures and was able to eat adequately within a few months. Although dentures are poor alternatives to healthy dentition,

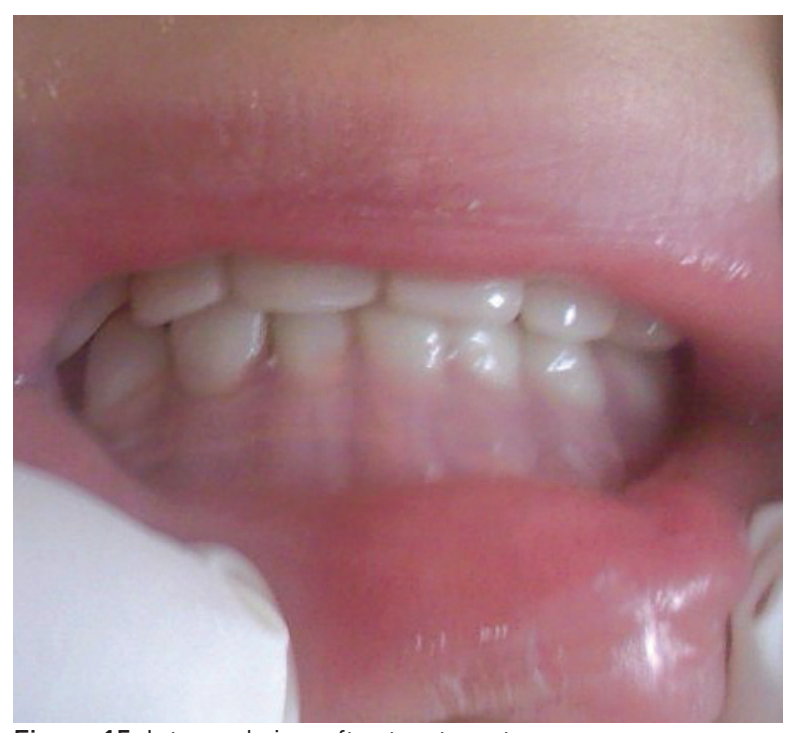

Figure 15. Intraoral view after treatment. they create conditions for the maintenance of a normal, satisfactory diet for the child. This is very important, considering that the establishment of lifelong dietary patterns occurs during childhood. ${ }^{2}$ Our observations in case 2 comply with this view.

Dental prostheses may also improve the tone of the muscles of mastication and may compensate for the reduced vertical dimension. ${ }^{10}$ Difficulty with mastication has been referred to as a major problem arising from loss of teeth. ${ }^{3}$ As seen in both cases, the facial profile and expression improved significantly with complete dentures; in addition, mastication and dietary patterns also improved.

Learning and reinforcement of articulation is known to continue until 8 years of age. ${ }^{3}$ Therefore, complete agenesis of primary teeth may result in speech abnormalities, as is seen in both cases. Regarding the dental treatment, it was possible to improve speech and communication skills and, as a consequence. Thereby, a higher self-esteem and better social acceptance was promoted with the establishment of complete dentures.

These case reports highlight the importance of accurate treatment planning as well as the influence of anodontia on the diagnosis of ectodermal dysplasia. Since the oral rehabilitation of these cases is often difficult, particularly in pediatric patients, treatment should be administered by a multidisciplinary team involving pediatric dentistry, orthodontics, prosthodontics, and oral-maxillofacial surgery.

\section{CONCLUSIONS}

The clinical manifestations of ectodermal dysplasia cause considerable social problems in individuals affected by the condition. In this case report, the prosthetic rehabilitation of 2 young boys with anhidrotic ectodermal dysplasia associated with severe anodontia was described. Since oligodontia or complete anodontia leads to atrophy of the alveolar bone, prosthetic treatment is of great value to these patients from functional, psychological, and psychosocial standpoints.

\section{REFERENCES}

1. Nunn JH, Carter NE, Gillgrass TJ, Hobson RS, Jepson NJ, Meechan JG, et al. The interdisciplinary management of hypodontia: background and role of paediatric dentistry. $\mathrm{Br}$ Dent $J$ 2003;194:245-251. 
2. Tarjan I, Gabris K, Rozsa N. Early prosthetic treatment of patients with ectodermal dysplasia: a clinical report. $J$ Prosthet Dent 2005;93:419-424.

3. Vieira KA, Teixeira MS, Guirado CG, Gaviao MB. Prosthodontic treatment of hypohidrotic ectodermal dysplasia with complete anodontia: case report. Quintessence Int 2007;38:75-80.

4. Abadi B, Herren C. Clinical treatment of ectodermal dysplasia: a case report. Quintessence Int 2001;32:743-745.

5. Yavuz I, Ülkü SZ, Ünlü G, Kama JD, Kaya S, Adıgüzel O, Kaya FA, Tümen EC, Zortuk M, Bahsi E, Arslanoḡlu Z. Ectodermal dysplasia: clinical diagnosis. Int Dent Med Disorders 2008;1:1-10.

6. Paschos E, Huth KC, Hickel R. Clinical management of hypohidrotic ectodermal dysplasia with anodontia: case report. J Clin Pediatr Dent 2002;27:5-8.

7. Imirzalioglu P, Uckan S, Haydar SG. Surgical and prosthodontic treatment alternatives for children and adolescents with ectodermal dysplasia: a clinical report. J Prosthet Dent 2002;88:569-572.

8. Lo Muzio L, Bucci P, Carile F, Riccitiello F, Scotti C, Coccia $E$, et al. Prosthetic rehabilitation of a child affected from anhydrotic ectodermal dysplasia: a case report. J Contemp Dent Pract 2005;6:120-126.

9. Adıgüzel O, Kaya S, Yavuz İ, Atakul F. Oral findings of ectodermal dysplasia and literature review. Int Dent Med Disorders 2008;1:43-49.

10. Itthagarun A, King NM. Ectodermal dysplasia: a review and case report. Quintessence Int 1997;28:595-602.

11. Lowry RB, Robinson GC, Miller JR. Hereditary ectodermal dysplasia. Symptoms, inheritance patterns, differential diagnosis, management. Clin Paediatric 1966;5:395-402.

12. Rad AS, Siadat H, Monzavi A, Mangoli AA. Full mouth rehabilitation of a hypohidrotic ectodermal dysplasia patient with dental implants: a clinical report. J Prosthodont 2007;16:209-213.

13. Murdock S, Lee JY, Guckes A, Wright JT. A costs analysis of dental treatment for ectodermal dysplasia. J Am Dent Assoc 2005; 136:1273-1276.

14. Hummel P, Guddack S. Psychosocial stress and adaptive functioning in children and adolescents suffering from hypohidrotic ectodermal dysplasia. Pediatr Dermatol 1997; 14:180-185.

15. Till MJ, Marques AP. Ectodermal dysplasia: treatment considerations and case reports. Northwest Dent 1992;71:2528. 